\title{
Short-Term Wind Power Interval Forecasting Based on an EEMD-RT-RVM Model
}

\author{
Haixiang Zang, ${ }^{1}$ Lei Fan, ${ }^{2}$ Mian Guo, ${ }^{1}$ Zhinong Wei, ${ }^{1}$ Guoqiang Sun, ${ }^{1}$ and Li Zhang ${ }^{1}$ \\ ${ }^{1}$ College of Energy and Electrical Engineering, Hohai University, Nanjing 211100, China \\ ${ }^{2}$ State Grid Chang Zhou Power Supply Company, Changzhou 213000, China
}

Correspondence should be addressed to Haixiang Zang; zanghaixiang@hhu.edu.cn

Received 3 June 2016; Revised 6 October 2016; Accepted 25 October 2016

Academic Editor: Caroline Draxl

Copyright (c) 2016 Haixiang Zang et al. This is an open access article distributed under the Creative Commons Attribution License, which permits unrestricted use, distribution, and reproduction in any medium, provided the original work is properly cited.

\begin{abstract}
Accurate short-term wind power forecasting is important for improving the security and economic success of power grids. Existing wind power forecasting methods are mostly types of deterministic point forecasting. Deterministic point forecasting is vulnerable to forecasting errors and cannot effectively deal with the random nature of wind power. In order to solve the above problems, we propose a short-term wind power interval forecasting model based on ensemble empirical mode decomposition (EEMD), runs test (RT), and relevance vector machine (RVM). First, in order to reduce the complexity of data, the original wind power sequence is decomposed into a plurality of intrinsic mode function (IMF) components and residual (RES) component by using EEMD. Next, we use the RT method to reconstruct the components and obtain three new components characterized by the fine-to-coarse order. Finally, we obtain the overall forecasting results (with preestablished confidence levels) by superimposing the forecasting results of each new component. Our results show that, compared with existing methods, our proposed short-term interval forecasting method has less forecasting errors, narrower interval widths, and larger interval coverage percentages. Ultimately, our forecasting model is more suitable for engineering applications and other forecasting methods for new energy.
\end{abstract}

\section{Introduction}

Industrialization practices are rapidly depleting fossil fuel reserves. Moreover, widespread use of fossil fuels produces large amounts of greenhouse gases and dust particles, both of which have significant negative effects on human society and the environment [1-3]. In order to address the energy crisis and alleviate environmental pressures, many countries are researching and utilizing forms of renewable energy [4-6]. Wind power has become especially prominent in the field of renewable clean energy because it is pollution-free, reserverich, and readily renewable [5]. Continuous improvements in wind power technology have led to an increase in the number of wind-powered grids. However, wind power is also random and volatile, and any serious power disturbances can affect the safety and stability of wind-powered grids. As such, accurate wind power forecasting is necessary for creating reasonable generation plans and system backup arrangements [7-9]. Ultimately, the key to increasing the number of wind-powered grids is to improve the wind power penetration limit of power grids.

Recent research and studies have greatly improved shortterm wind power forecasting. Many methods, such as the time series method [7, 10-12], Kalman filtering [13], model structure selection [14], fuzzy logic method [15], the artificial neural networks (ANNs) method [16-19], wavelet transformation [20], and support vector machines [21] have been utilized for wind power forecasting. Additionally, other combined methods have become popular in recent years [22-25].

The stochastic volatility of natural wind and its effects on wind-powered grids cannot be ignored. Interval forecasting can effectively reflect the uncertainties in the forecasting results. Deterministic point forecasting methods have some deficiencies in characterizing the randomness of actual wind power [26]. Therefore, it is necessary to establish a forecasting method that is capable of efficiently providing accurate information. If we can establish a forecasting method capable of providing accurate interval forecasting, we will better 
understand potential fluctuations in wind power, which will allow for the creation of standby arrangements for power systems [27, 28]. Compared to deterministic point forecasting, interval forecasting is still in its infancy. Interval forecasting has become more studied in recent years, and various interval forecasting methods have been proposed. The existing interval forecasting methods include the bootstrap method, the quantile regression method, the mixed structure interval method, and the probability interval forecasting method. The bootstrap method [29] constructs a sample set based on computer resampling technology, which requires a large amount of original data processing, and consumes much time and computation. The quantile regression method [30-32] utilizes a rigorous theoretical background and yields reliable results; however, it requires a predetermined regression model and subsites, complicated calculations, and its forecasting accuracy is significantly reduced when the predicting samples increase. The mixed structure interval method $[33,34]$ is usually based on point forecasting results, with the interval result being determined by the calculation of coefficients and error analysis. The probability interval forecasting method [35-38] constructs a load distribution so as to directly obtain the expectations and forecasting distribution of the load. The forecasting interval can then be drawn under an arbitrarily determined confidence level.

In order to establish a more simplistic and accurate shortterm interval forecasting method, we propose a combined model based on ensemble empirical mode decomposition (EEMD) and a relevance vector machine (RVM). As part of our model, we use the framework of the probabilistic interval forecasting method, specifically the runs test reconstruction method, in order to achieve short-term interval wind power forecasts. First, RVM (a relatively new machine learning algorithm) combines the Markov Native theory, the Bayesian theorem, and the autorelated decisions a priori and maximum likelihood theory. Compared with ANN and SVM, not only does RVM have the advantages of higher model sparsity, fewer kernel function limits, and stronger generation ability, it can also obtain probabilistic forecasting results within the framework of the Bayesian theory and the statistical learning theory [39]. Second, in order to improve the forecasting accuracy of our model and narrow its width of interval range, we altered and improved two aspects of data decomposition preprocessing, as well as model parameter optimization. The EEMD is used to decompose the original wind power sequence into a series of IMF components and RES component in order to reduce its complexity. The RT method is then used to reconstruct these IMF components and RES component into a trend component, a detailed component, and a random component. Finally, a combination of the typical local kernel of the RBF kernel and the global kernel of the polynomial kernel is used to obtain better forecasting results.

Our proposed EEMD-RT-RVM model is used to achieve the one-point-ahead $15 \mathrm{~min}$ ahead short-term wind power interval forecasting. We used a variety of evaluation indexes to conduct comparative analyses and impact assessments for both our proposed model and other existing models.
The results show that our combined model obtains higher forecasting accuracy and narrower interval widths than other existing methods. As such, our proposed model has high research significance and practical value.

\section{Methodologies}

2.1. Empirical Mode Decomposition (EMD). EMD is an efficient signal decomposition method that does not rely on any predefined basis function. The EMD reflects the dynamics of signals more accurately than other models. The modes extracted by the EMD, named the intrinsic mode functions (IMF), are defined by the following criteria: (1) the number of extrema and zero crossings must be equal or differ by no more than one and (2) the local mean of the envelope defined by the local maxima and local minima must be zero [40, 41]. These two criteria ensure that each IMF has a physically meaningful phase definition; however, the time invariant frequency does not necessarily have a meaningful phase definition.

Given a signal $d(t)$, the EMD algorithm can be summarized as follows.

Step 1. Initialize the loop variable $i=1, d_{1}(t)=d(t)$, where $d(t)$ is the given original data.

Step 2. Initialize the loop variable $j=1, g_{1}(t)=d_{1}(t)$.

Step 3. Find out all the local minima and maxima of $d(t)$, and interpolate between the local minima and maxima, respectively, in order to get an upper envelope $u_{j}(t)$ and a lower envelope $v_{j}(t)$. The mean value of these envelopes is described as

$$
m_{j}(t)=\frac{u_{j}(t)+v_{j}(t)}{2} .
$$

Next, compute the minis of the original data and the envelope mean value as

$$
h_{j}(t)=g_{j}(t)-m_{j}(t) .
$$

Step 4. Check whether $h_{j}(t)$ satisfies the two criteria for an IMF (as defined above). If it is not satisfied, make $j=j+1$, $g_{j}(t)=h_{j}(t)$, and repeat Step 3. If it is satisfied, the first IMF can be given as

$$
c_{i}(t)=h_{j}(t) .
$$

The residual can be computed by

$$
r_{i}(t)=d_{i}(t)-c_{i}(t) .
$$

Step 5. Treat $r_{j}(t)$ as a new signal and repeat Steps 1-4 (in order to find more IMFs) until the residual is a constant or a monotonic function. Finally, the given $d(t)$ can be decomposed into $n$ IMFs and a final residual $r_{n}(t)$ as follows:

$$
d(t)=\sum_{i=1}^{n} c_{i}(t)+r_{n}(t)
$$


2.2. Ensemble Empirical Mode Decomposition (EEMD). Mode mixing is the most significant drawback of EMD. Mode mixing implies either a single IMF consisting of signals of dramatically disparate scales or a signal of the same scale appearing in different IMF components. This causes intermittency when analyzing signals.

In order to solve the problem of mode mixing in EMD, $\mathrm{Wu}$ and Huang proposed a new noise-assisted data analysis method called ensemble empirical mode decomposition (EEMD) [42]. The EEMD method utilizes recent studies on white noise which showed that the EMD method is an effective self-adaptive dyadic filter bank when applied to white noise. The results demonstrate that noise can help data analysis in the EMD method [22, 43].

Two important parameters used in the EEMD method are (1) the amplitude $k$ of white noise and (2) the total repeat number $M$ of the EMD. At present, the determination of $k$ and $M$ is based on the structural characteristics of the data. Generally, the $M$ taken is 100 , and $k$ is chosen from a range of $0.05 \sim 0.5$. Based on previous tests, we set $M=100$ and $k=0.2$ in this paper. follows:

The specific steps of the EEMD can be described as

(1) Set the value of the amplitude $k$ and the total repeat number $M$.

(2) Add a white noise series to the signal.

(3) Decompose the signal with the added white noise into IMFs by using EMD.

(4) Repeat steps (2) and (3) using different levels of white noise each time and obtain corresponding IMF components of the decomposition. Calculate the mean of all the corresponding IMF components. Take the mean as the final result for each IMF. Calculate the mean of all the residual (RES) components and take the mean as the final result for the RES component:

$$
\begin{aligned}
& \overline{c_{i}}(t)=\sum_{m=1}^{M} \frac{c_{i \cdot m}(t)}{M}, \\
& \overline{r_{n}}(t)=\sum_{m=1}^{M} \frac{r_{n \cdot m}(t)}{M} .
\end{aligned}
$$

(5) Take the $\overline{c_{i}}(t)(i=1, \ldots, n)$ and $\overline{r_{n}}(t)$ as the IMF components and RES component, respectively.

2.3. Runs Test (RT). The runs test method [44] is defined in the following.

Assume the time series corresponds to $\mathrm{IMF}_{i}$ and RES as $\{X(t)\}_{t=1}^{N}$, where $i$ is the label of IMF, $t$ is label of samples, and $N$ is the total number of samples. The mean value of the samples is defined as

$$
\bar{X}=\frac{1}{N} \sum_{t=1}^{N} X(t)
$$

Then, the timing symbol $S_{i}$ can be defined as

$$
S_{i}=X(t)-\bar{X}= \begin{cases}1 & X(t)>\bar{X}, \\ 0 & X(t) \leq \bar{X}\end{cases}
$$

where $S_{i}$ consists of a series of statistically independent randomly arranged sequences of 0 and 1 .

Define each sequence with successive symbols ( 0 or 1$)$ as a runs test. The total runs test number of each $S_{i}$ can be used to detect the fluctuation of each component obtained by the EEMD. Next, the high and low runs test thresholds can be set according to the runs test, and the components decomposed by the EEMD will be reconstructed into three new components (with typical characteristics based on the fine-tocoarse order) [44]. This ensures the decomposition effect and significantly reduces the run time of the model. Moreover, the similar components are reconstructed, strengthening the inherent laws of these data, to improve the prediction accuracy.

2.4. Relevance Vector Machine (RVM). Compared with other forecasting algorithms, the RVM not only has high sparsity, less optimized parameters, flexible kernels, and strong generalization abilities, but also directly implements interval forecasting $[45,46]$. Therefore, in this study, the RVM is used to establish the interval forecasting model for the new components reconstructed by RT.

For a given set of input training samples $\left\{x_{n}\right\}_{n=1}^{N}$ and the corresponding output sets $\left\{y_{n}\right\}_{n=1}^{N}$, the relevance vector machine regression model can be defined as follows:

$$
y_{i}=\sum_{i=1}^{N} \omega_{i} K\left(x, x_{i}\right)+\omega_{0}+\varepsilon
$$

where $\varepsilon$ is the error of the independent sample (which follows the Gaussian distribution with the variance $\left.\sigma^{2}\right), \omega_{i}$ is the model weights, $K\left(x, x_{i}\right)$ is a nonlinear kernel function, $x$ is relevance vector, and $N$ is the length of the data.

In the RVM, a priori probability distribution for each model weight is given as

$$
p\left(\omega_{i}, \alpha_{i}\right)=\left\{\frac{\alpha_{i}}{2 \pi}\right\}^{1 / 2} \exp \left\{-\frac{1}{2} \alpha_{i} \omega_{i}^{2}\right\},
$$

where $\alpha_{i}$ is the hyperparameter of a priori distribution of model weight $\omega_{i}$.

Given a training sample set $\left\{x_{n}\right\}_{n=1}^{N}$, assume the target value $y_{i}$ is independent and the noise in the data follows the Gaussian distribution with the variance $\sigma^{2}$. Then, the likelihood function of the training sample set can be represented by

$$
\begin{aligned}
p\left(y \mid \omega, \sigma^{2}\right) & =\prod_{n=1}^{N} p\left(y_{i} \mid \omega, \sigma^{2}\right) \\
& =\left\{2 \pi \sigma^{2}\right\}^{-N / 2} \exp \left\{-\frac{\|y-\Phi \omega\|^{2}}{2 \sigma^{2}}\right\},
\end{aligned}
$$


TABLE 1: The runs value of each component.

\begin{tabular}{lccccccccc}
\hline Component & IMF1 & IMF2 & IMF3 & IMF4 & IMF5 & IMF6 & IMF7 & IMF8 & RES \\
\hline Runs value & 359 & 153 & 64 & 31 & 17 & 5 & 5 & 3 & 2 \\
\hline
\end{tabular}

TABLE 2: The composition of each new component.

\begin{tabular}{lccc}
\hline New component & Random component & Detailed component & Trend component \\
\hline EEMD component & IMF1, IMF2 & IMF3, IMF4, IMF5 & IMF6, IMF7, IMF8, RES \\
\hline
\end{tabular}

where $y=\left(y_{1}, \ldots, y_{n}\right)^{T}, \omega=\left(\omega_{0}, \omega_{1}, \ldots, \omega_{n}\right)^{T}$, and $\Phi$ is the design matrix given by

$$
\Phi=\left[\begin{array}{ccccc}
1 & K\left(x_{1}, x_{1}\right) & K\left(x_{1}, x_{2}\right) & \cdots & K\left(x_{1}, x_{N}\right) \\
1 & K\left(x_{2}, x_{1}\right) & K\left(x_{2}, x_{2}\right) & \cdots & K\left(x_{2}, x_{N}\right) \\
\vdots & \vdots & \vdots & \ddots & \vdots \\
1 & K\left(x_{N}, x_{1}\right) & K\left(x_{N}, x_{2}\right) & \cdots & K\left(x_{N}, x_{N}\right)
\end{array}\right] .
$$

Based on a priori probabilities distribution and the likelihood distribution, the posterior distribution over the weight forms Bayes rule and can be written as

$$
\begin{gathered}
p\left(\omega \mid y, \alpha, \sigma^{2}\right)=\frac{p\left(y \mid \omega, \sigma^{2}\right) p(\omega \mid \alpha)}{p\left(y \mid \alpha, \sigma^{2}\right)} \\
=\{2 \pi\}^{-(N+1) / 2}|\Sigma|^{-1 / 2} \\
\cdot \exp \left\{-\frac{1}{2}(\omega-\mu)^{T} \Sigma^{-1}(\omega-\mu)\right\},
\end{gathered}
$$

where $\Sigma=\left(\sigma^{-2} \Phi^{T} \Phi+A\right)^{-1} ; \mu=\sigma^{-2} \Sigma \Phi^{T} y ; A=\operatorname{diag}\left(a_{0}\right.$, $\left.a_{1}, \ldots, a_{N}\right)$.

The marginal likelihood distribution of the hyperparameters can be obtained by

$$
p\left(y \mid \alpha, \sigma^{2}\right)=\{2 \pi\}^{-N / 2}|\Omega|^{-1 / 2} \exp \left\{-\frac{y^{T} \Omega^{-1} y}{2}\right\},
$$

where $\Omega=\sigma^{2} I+\Phi A^{-1} \Phi^{T}$.

Finally, the hyperparameter $\alpha$ and the variance $\sigma^{2}$ can be estimated by using the maximum likelihood algorithm.

If the input value is $x_{i}^{*}$, then the corresponding output probability distribution obeys the Gaussian distribution, and the corresponding forecasting value can be derived by

$$
\begin{aligned}
& y_{*}=\varphi\left(x_{i}^{*}\right) \mu, \\
& \sigma_{*}^{2}=\sigma_{M P}^{2}+\varphi\left(x_{i}^{*}\right)^{T} \Sigma \varphi\left(x_{i}^{*}\right) .
\end{aligned}
$$

The RVM model can give both the mean value and the variance. As such, this model reflects the uncertainty of forecasting results and provides accurate interval forecasting (within the range of certain confidence levels).

Under the confidence level of $\alpha$, the interval forecasting results are as follows:

$$
[\mathrm{Lb}, \mathrm{Ub}]=\left[y_{*}-z_{\alpha / 2} \sigma_{*}, y_{*}+z_{\alpha / 2} \sigma_{*}\right]
$$

where $\mathrm{Lb}$ denotes the lower bound of the forecasting value and $\mathrm{Ub}$ denotes the upper bound of the forecasting value.

\section{Model Construction}

3.1. Using EEMD to Decompose an Original Wind Power Sequence. In order to verify the effectiveness of the forecasting model, the whole year wind power sequence (96 point one day) obtained from a wind farm in Jiangsu province is used as the research object. The installed capacity of this wind farm in Jiangsu province is $49.5 \mathrm{MW}$, which contains 33 wind turbines. In this study, actual wind power data ( 5 days ago) is taken as the training sample. Then we establish wind power interval forecasting model for the next day in advance 15 minutes' forecast.

As is shown in Figure 1(a), the actual wind power is random and volatile. In order to improve the forecasting effect, it is necessary to reduce the complexity of the data. Compared with other decomposition algorithms, the EEMD exhibits better noise robustness and decomposing effects. In this study, we use the EEMD to decompose actual wind power and to establish specific components, in which the periodicity, randomness, and the trends of the actual wind power can be clearly seen in the components. The decomposition results of EEMD are shown in Figure 1.

3.2. Using RT to Reconstruct the New Components. By the definition of RT, the RT value is greater, the volatility of time series is stronger, the RT value is closer, and the overall trend of time series is more similar. The RT values of each component (in Figure 1) are calculated and shown in Table 1. From Figure 1 and Table 1, the RT values of IMF1 and IMF2 are significantly large and relatively close, while the RT values of IMF6-IMF8 and RES are too small and very close. Also, the RT values of IMF3-IMF5 are found between the two. This shows that the dispersion of IMF1 and IMF2 series is strong, while the general trend of IMF6-IMF8 and RES is similar. Moreover, the fluctuation trend of IMF3-IMF5 is between the two. Based on the above analyses and studies, we set the high runs test threshold as 100 and the low runs test threshold as 10. The composition of the three new components is shown in Table 2.

The trend graph of the new components after reconstruction is shown in Figure 2. It is evident from Figure 2 that the trend component, the detailed component, and the random component each have typical features. The trend component roughly reflects the overall fluctuation of the original data; the detailed component characterizes the details of the fluctuations of the original data; and the random component represents the fluctuations caused by other factors that cannot 


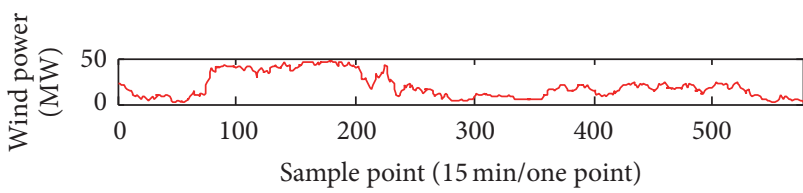

(a)

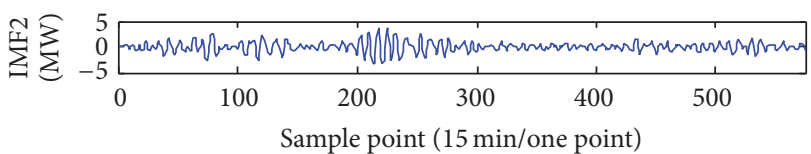

(c)

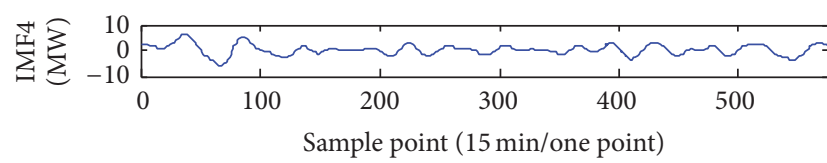

(e)

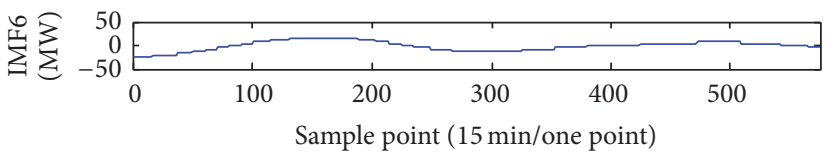

(g)

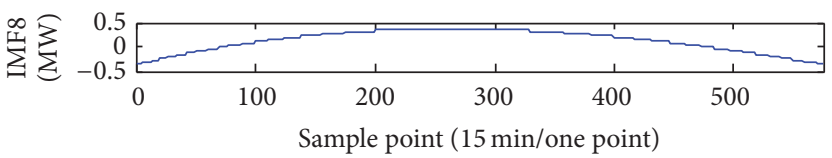

(i)

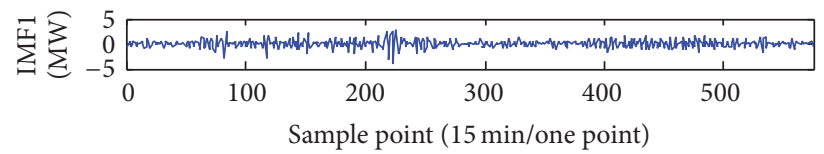

(b)

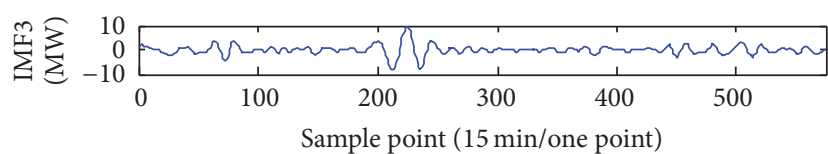

(d)

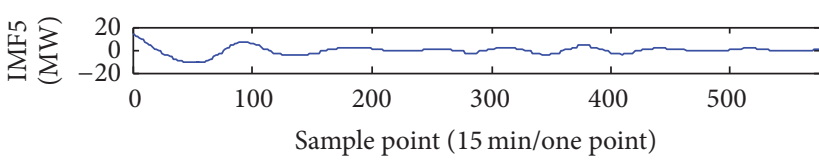

(f)

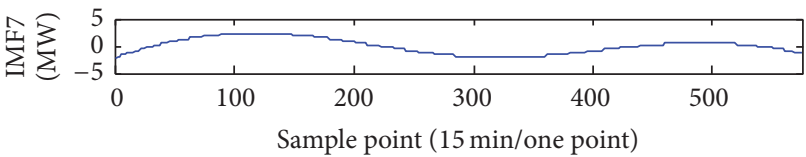

(h)

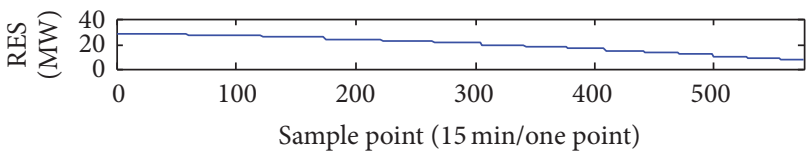

(j)

FIGURE 1: The decomposition results of the EEMD.

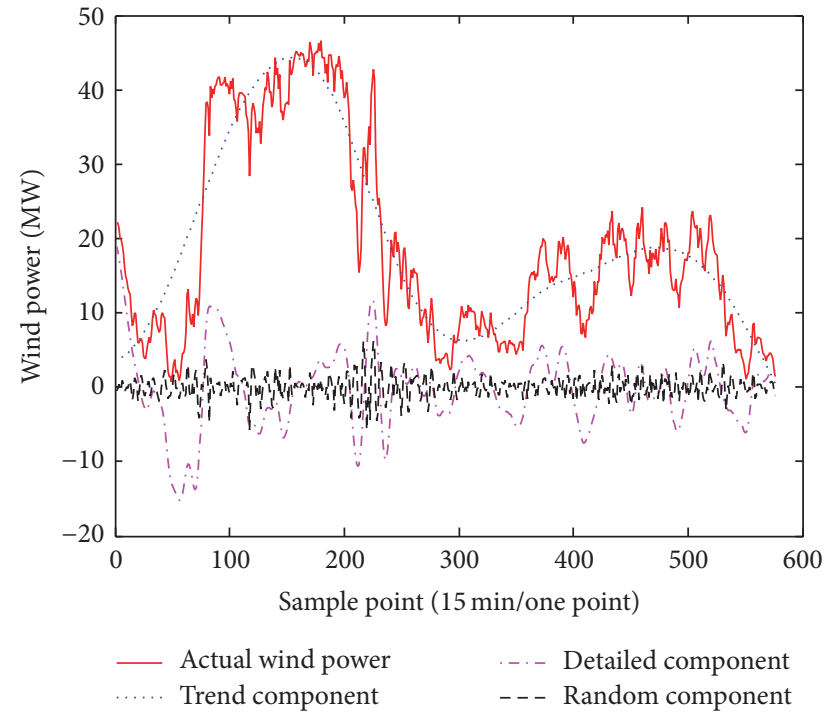

FIgURE 2: Trend graph of each new component.

to be explicitly described. All three of the components meet the composition standard of actual wind power.

In order to further simplify the calculation and narrow the forecasting interval, we established a point forecasting for the trend component and obtained the interval forecasting for the detailed component and the random component. We obtained the overall optimal interval forecasting results (under a certain confidence level) from the superposition of each component's forecasting results.

3.3. Sample Data Normalization. Since poor and missing data affect forecasting accuracy, it is necessary to pretreat load data obtained from measurements. In this study, we primarily used transverse and longitudinal comparisons methods for data pretreatment. In addition, we used the normalization method in order to simplify the calculation and standardization of loads, prices, and weather data (a necessary measure since the input variables have different units and values). By doing so, the value of the data can be limited to $[0,1]$. The specific calculation formula is

$$
\widehat{x}(i)=\frac{x(i)-x_{\min }}{x_{\max }-x_{\min }},
$$

where $\widehat{x}(i)$ is the normalized value of the data and $x_{\max }$ and $x_{\min }$ represent the maximum value and the minimum value of the data, respectively.

3.4. Kernel Function Determination of RVM. RVM is a pattern recognition and regression forecasting method based 
on kernel functions. The kernels implement nonlinear transformations among a plurality of feature spaces. The basic idea of hybrid kernels is to combine a plurality of kernels with different characteristics (in a certain proportion) in order to ensure that the combined kernel function has better performance. Importantly, RVM is less limited in kernel function selection. Moreover, RBF kernels are well-suited to solving local fluctuations, while polynomial kernels are wellsuited to dealing with global fluctuations. A combination of typical RBF local kernels and the global kernels (of polynomial kernels) is used for improved short-term wind power interval forecasting. The hybrid kernel is shown as follows:

$$
\begin{aligned}
& K\left(x, x_{i}\right)=\lambda G\left(x, x_{i}\right)+(1-\lambda) P\left(x, x_{i}\right), \\
& G\left(x, x_{i}\right)=\exp \left\{-\frac{\left\|x-x_{i}\right\|^{2}}{\sigma^{2}}\right\} \\
& P\left(x, x_{i}\right)=\left(x \cdot x_{i}\right)=\left(x \cdot x_{i}+1\right)^{2},
\end{aligned}
$$

where $G\left(x, x_{i}\right)$ is the RBF kernel; $P\left(x, x_{i}\right)$ is the binomial kernel function; $\lambda$ is the weight of the kernel function; $\delta$ is the kernel width; and $\lambda$ and $\delta$ are the parameters to be optimized. We employed the grid search method in order to obtain the optimal values of $\lambda$ and $\delta$.

3.5. Evaluation Indexes of the Model. There are many indexes used to evaluate the errors of point forecasting results, such as APE (absolute percentage error), MAPE (mean absolute percentage error), and RMSE (root mean square error) [47-49]. The smaller the error, the higher the forecasting accuracy. The assessment methods of interval forecasting differ from point forecasting (except when using the MAPE index). Other indexes used to evaluate the efforts of interval forecasting results are FICP (forecasting interval coverage percentage) [50] and FIAW (forecasting interval average width). The definitions of these methods are as follows.

\section{(1) MAPE}

$$
\text { MAPE }=\frac{1}{N} \sum_{i=1}^{N}\left|\frac{\bar{y}_{i}^{*}-y_{i}}{y_{i}}\right| \times 100 \%,
$$

where $\bar{y}_{i}^{*}$ represents the forecasting result of the $i$ th forecasting sample; $y_{i}$ represents the true value of the $i$ th forecasting sample; and $N$ represents the number of the sample. MAPE is used to evaluate the error between the expected forecasting value and the actual value. The smaller the value, the higher the forecasting accuracy.

\section{(2) FICP}

$$
\operatorname{FICP}^{(1-\alpha)}=\frac{1}{N} \xi^{(1-\alpha)} \times 100 \%,
$$

where FICP ${ }^{(1-\alpha)}$ represents the interval coverage; and $\xi^{(1-\alpha)}$ is the number of the actual value falling within the confidence interval (at the confidence level). The index of FICP evaluates

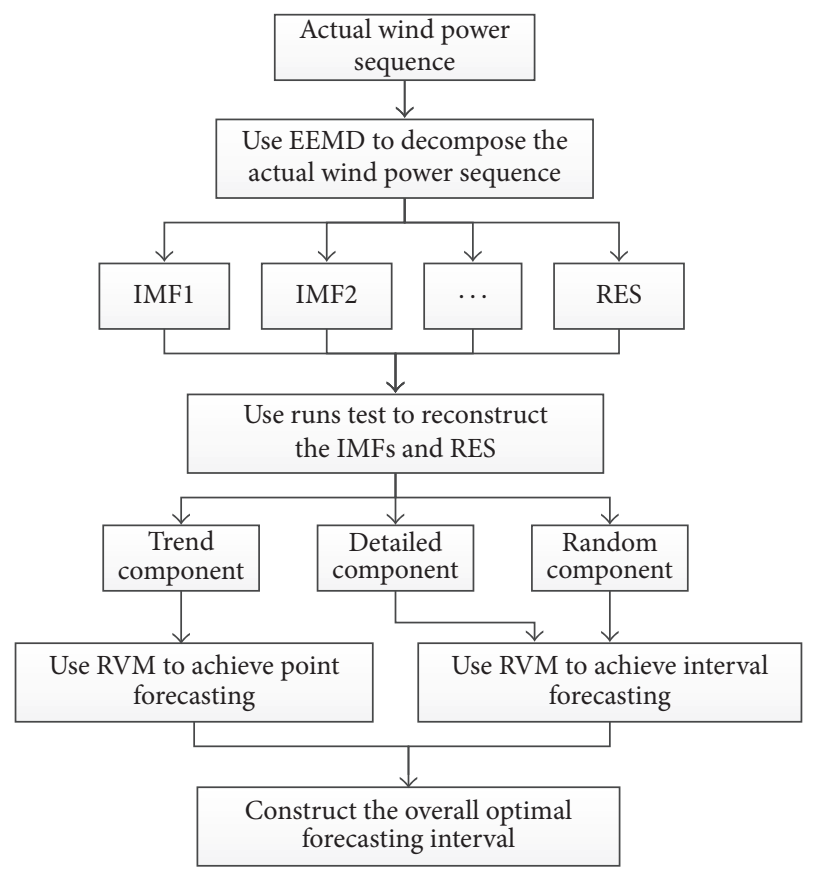

FIGURE 3: Flow chart of the short-term wind power interval forecasting model using EEMD-RT-RVM.

the credibility of the interval. The greater its value, the higher its credibility.

(3) FIAW

$$
\operatorname{FIAW}^{(1-\alpha)}=\frac{1}{N} \sum_{i=1}^{N} \frac{U\left(x_{i}\right)-L\left(x_{i}\right)}{y_{i}}
$$

where FIAW $^{(1-\alpha)}$ represents the average width of the confidence interval under the level $1-\alpha ; U\left(x_{i}\right)$ and $L\left(x_{i}\right)$ are, respectively, the upper and lower bounds of the $i$ th forecasting sample; and $y_{i}$ refers to the actual value of the $i$ th forecasting sample. The index of FIAW is based on its ability to evaluate the uncertain degree of the forecasting results.

3.6. Overall Procedures of the EEMD-RT-RVM Model. In this study, we propose a short-term wind power interval forecasting method based on the EEMD-RT-RVM model. The flow chart of our proposed forecasting model is shown in Figure 3.

\section{Analysis Results of the Demonstration}

In order to verify the interval forecasting effects of an EEMDRT-RVM model that uses different confidence levels, we chose to use confidence levels of $90 \%$ and $60 \%$ in our example. The interval forecasting results are shown in Figures 4 and 5. The indexes of MAPE, FICP, and FIAW are used to assess the effects of the interval forecasting. Table 3 shows portions of the forecasting results and the indicator analysis results. 


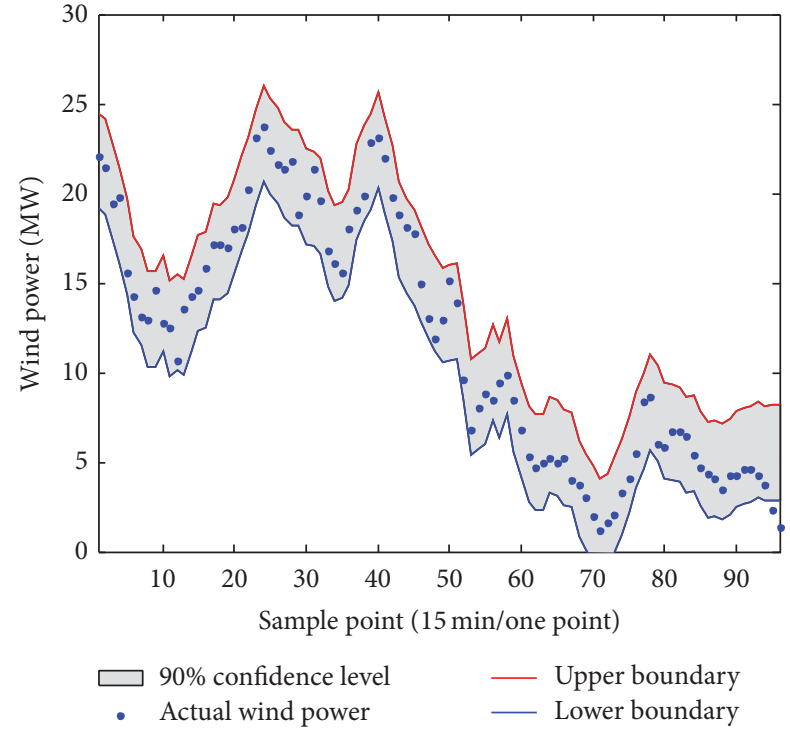

Figure 4: Interval forecasting results with a $90 \%$ confidence level.

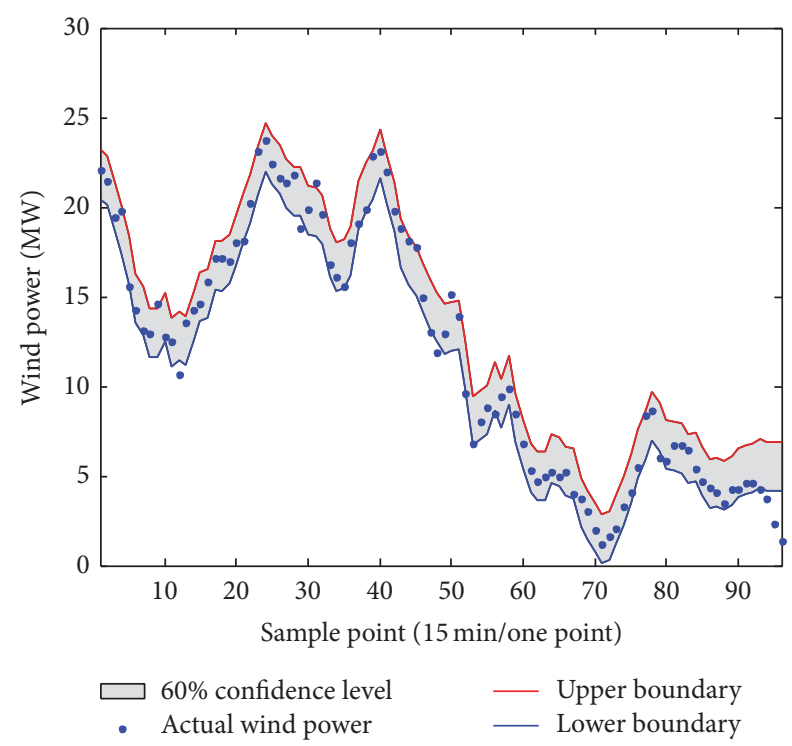

Figure 5: Interval forecasting results with a $60 \%$ confidence level.

To prove the superiority of our model, we used the same wind power to obtain short-term interval forecasting from the RVM model, the EMD-RVM model, and the EEMDRVM model. We used the indexes of MAPE, FICP, and FIAW, and their running times to assess their effects on interval forecasting. Table 4 shows the comparison results of the models (under the $90 \%$ confidence level).

Moreover, in order to further evaluate the adaptability of this proposed model, the wind power data of the actual wind farm in the other days of different seasons are chosen for the research. For example, the dates of February 12, July 22, October 15, and December 17, 2009, are chosen randomly.
TABLE 3: Interval forecasting results of the EEMD-RT-RVM model.

\begin{tabular}{lccc}
\hline & $\begin{array}{c}90 \% \\
\text { confidence } \\
\text { level }\end{array}$ & $\begin{array}{c}60 \% \\
\text { confidence } \\
\text { level }\end{array}$ & $\begin{array}{c}\text { Actual wind } \\
\text { power/MW }\end{array}$ \\
\hline MAPE & $5.77 \%$ & $5.77 \%$ & - \\
FICP & $97.92 \%$ & $83.33 \%$ & - \\
FIAW & 0.7836 & 0.4009 & - \\
$0: 00$ & {$[19.13,24.47]$} & {$[20.44,23.16]$} & 23.16 \\
$0: 15$ & {$[18.83,24.16]$} & {$[20.13,22.85]$} & 22.85 \\
$0: 30$ & {$[17.34,22.67]$} & {$[18.64,21.37]$} & 21.37 \\
$0: 45$ & {$[16.09,21.42]$} & {$[17.39,20.12]$} & 20.12 \\
$\vdots$ & $\vdots$ & $\vdots$ & $\vdots$ \\
$23: 30$ & {$[2.87,8.21]$} & {$[4.18,6.90]$} & 2.36 \\
$23: 45$ & {$[2.86,8.19]$} & {$[4.16,6.88]$} & 1.39 \\
\hline
\end{tabular}

TABle 4: Comparison of the forecasting effects among the six models.

\begin{tabular}{lcccc}
\hline Forecasting model & MAPE & FICP & FIAW & Running time \\
\hline ELM & $12.08 \%$ & $88.37 \%$ & 0.8435 & $7 \mathrm{~s}$ \\
RVM & $11.79 \%$ & $87.50 \%$ & 0.7782 & $9 \mathrm{~s}$ \\
Wavelet-RVM & $9.87 \%$ & $91.36 \%$ & 0.8764 & $64 \mathrm{~s}$ \\
EMD-RVM & $8.15 \%$ & $92.77 \%$ & 0.8402 & $86 \mathrm{~s}$ \\
EEMD-RVM & $6.96 \%$ & $95.64 \%$ & 0.8038 & $97 \mathrm{~s}$ \\
EEMD-RT-RVM & $5.77 \%$ & $97.92 \%$ & 0.7836 & $38 \mathrm{~s}$ \\
\hline
\end{tabular}

TABLE 5: Comparison of index results among four different days.

\begin{tabular}{lccc}
\hline Forecast day & MAPE & FICP & FIAW \\
\hline February 12, 2009 & $5.23 \%$ & $87.1 \%$ & 0.1559 \\
July 22, 2009 & $6.48 \%$ & $89.37 \%$ & 0.2549 \\
October 15, 2009 & $6.27 \%$ & $92.71 \%$ & 0.3887 \\
December 17, 2009 & $6.06 \%$ & $97.92 \%$ & 0.3004 \\
\hline
\end{tabular}

Based on the time scale of the original wind power data, the $15 \mathrm{~min}$ ahead short-term wind interval forecasting results under the $90 \%$ confidence level for these days are shown in Figure 6. The indicator analysis results with MAPE, FICP, and FIAW are organized in Table 5. In Figure 6, the interval width is narrower in July than October. It means the data fluctuation of October is stronger than the data of July. It is found in Figure 6 and Table 5 that the MAPE indicator can reflect the effectiveness of the proposal method. The smaller the MAPE, the better the forecasting accuracy, illustrating the forecasting expected value is closer to actual result. Further, the MAPE of different days are all within $6.5 \%$ and meet the actual project requirements. The interval width becomes narrower with the smaller FIAW value due to the better MAPE results and this decreases the model uncertainty. Meanwhile, the credibility of forecasting results may reduce with the smaller FICP value. 


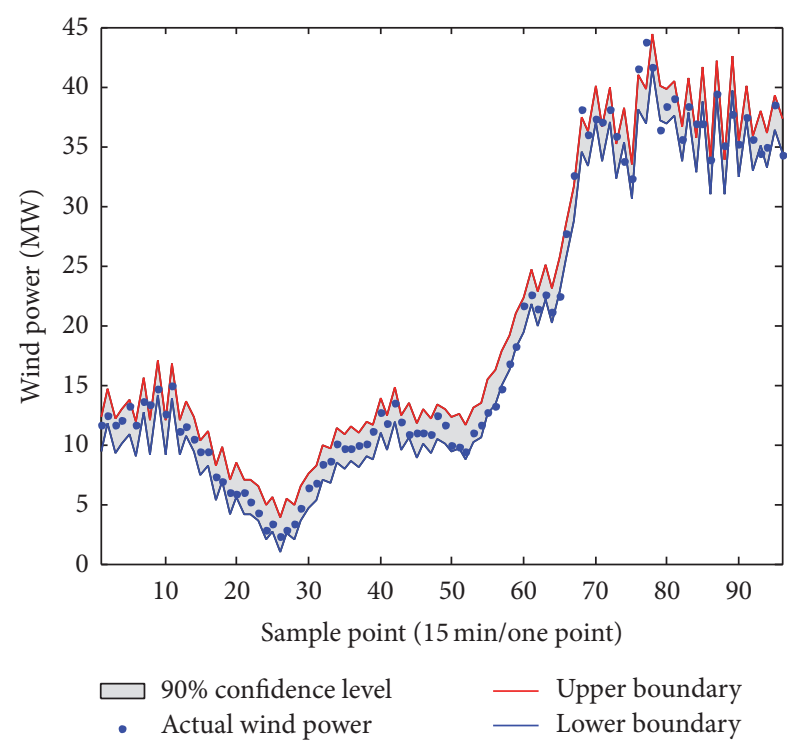

(a) February 12, 2009

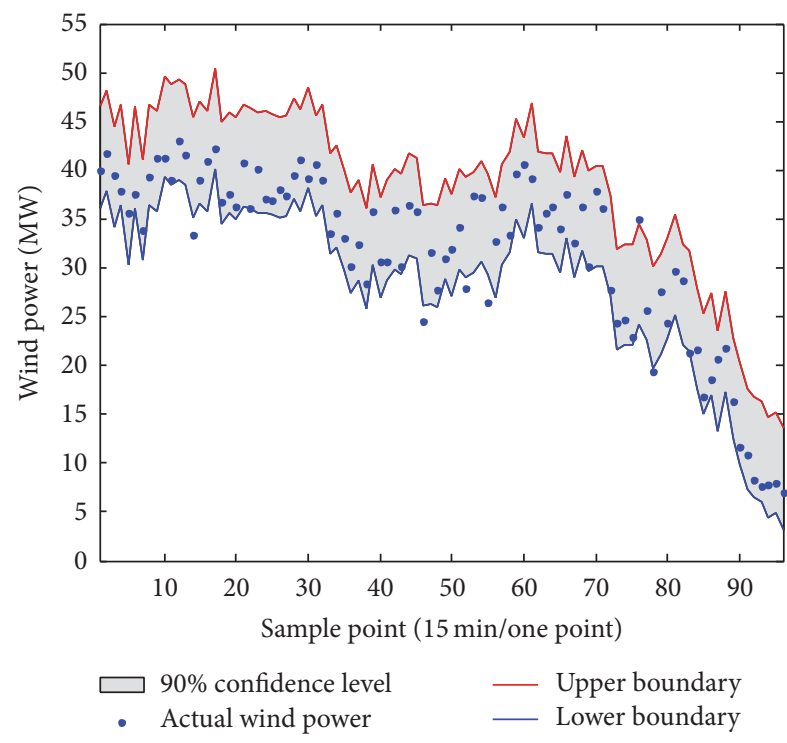

(c) October 15, 2009

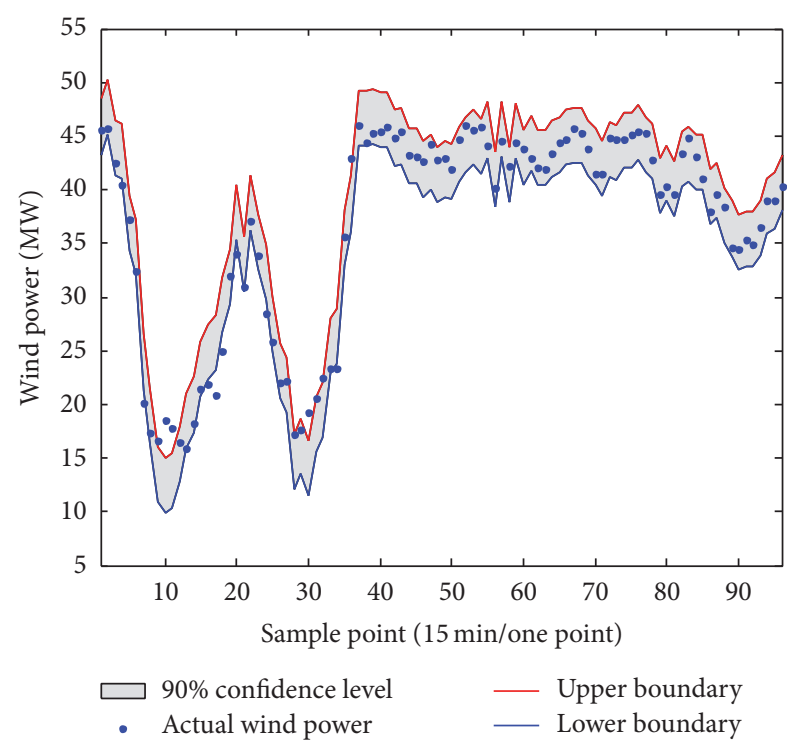

(b) July 22, 2009

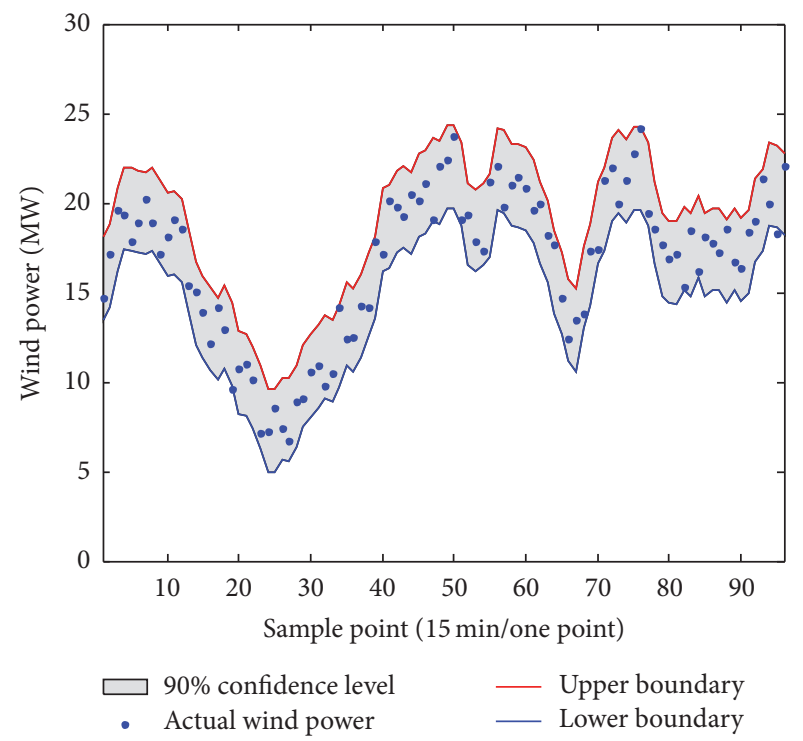

(d) December 17, 2009

FIGURE 6: Interval forecasting results of four days for the wind farm.

Based on the above analysis, we drew the following five conclusions: (1) the forecasting results of our proposed model effectively follow the actual wind power value, and the fluctuations are consistent with changes in actual wind power. (2) Most of the actual wind power falls within the forecasting interval with confidence levels of $90 \%$ and $60 \%$; however, the number of forecasts falling outside the forecasting interval of the $60 \%$ confidence level is significantly larger than those falling outside the interval of the $90 \%$ confidence level. This accurately depicts the characteristics of the actual situation and reflects the effectiveness of our interval forecasting results. (3) The interval width of the $90 \%$ confidence level is significantly greater than that of the $60 \%$ confidence level. Decreases in the confidence level also decrease the interval width and the interval coverage. (4) Overall, our proposed model had minimum forecasting errors, narrower interval widths, and higher interval coverages than any of the other models. (5) The EEMD has a better theoretical foundation and noise robustness than our model; however, we overcome this weakness by using the mode mixing phenomenon of EMD. Moreover, the use of runs tests uncovers the correlation among the components and reduces the complexity of our model (which contributes to improved forecasting effects and enhanced running efficiency).

In summary, our EEMD-RT-RVM model had the better performance results in forecasting short-term wind power interval. Furthermore, our model is applicable to other practical engineering applications. 


\section{Conclusions}

Owing to the volatility and randomness of the nature wind, the deterministic wind power forecasting is a difficult and complex task. In this study, we proposed an EEMD-RT-RVM model to achieve more accurate short-term interval wind power forecasting. The EEMD was used to decompose wind power sequences into IMF components and RES component, which reduced the inherent volatility of the wind power sequences. We then used runs tests to reconstruct new components. Overall, our methods improved the forecasting performance and enhanced running efficiency. The actual wind power data from November 20, 2009, to November 25, 2009 ( $15 \mathrm{~min} /$ one point), are used to verify the effectiveness and superiority of the proposed VMD-RT-RVM model, and quantitative evaluation is conducted based on comprehensive error evaluation criteria and interval evaluation criteria. Simulation results and analysis demonstrate that the volatility and randomness of wind power are reduced by the EEMD method, and the proposed EEMD-RT-RVM model performs better than conventional single models and other combined models. Our proposed EEMD-RT-RVM model not only improves forecasting accuracy, but also significantly reduces the width of the forecasting interval (under the premise of guaranteed interval coverage). Ultimately, our model is suitable for numerous practical applications, and it also serves as a good reference value for the output forecasting of other new energy sources.

\section{Competing Interests}

The authors declare that there is no conflict of interests regarding the publication of this paper.

\section{Acknowledgments}

The research is financially supported by National Natural Science Foundation of China (Program no. 51507052), China Postdoctoral Science Foundation (2015M571653), the 111 Project (B14022), and the Fundamental Research Funds for the Central Universities (2015B02714, 2016B20914).

\section{References}

[1] S. Kheradmand, O. Nematollahi, and A. R. Ayoobi, "Clearness index predicting using an integrated artificial neural network (ANN) approach," Renewable and Sustainable Energy Reviews, vol. 58, pp. 1357-1365, 2016.

[2] A. Teke, H. B. Yıldırım, and Ö. Çelik, "Evaluation and performance comparison of different models for the estimation of solar radiation," Renewable and Sustainable Energy Reviews, vol. 50, pp. 1097-1107, 2015.

[3] F. Onea, A. Raileanu, and E. Rusu, "Evaluation of the wind energy potential in the coastal environment of two enclosed seas," Advances in Meteorology, vol. 2015, Article ID 808617, 14 pages, 2015.

[4] Y. S. Xue, C. Yu, K. Li et al., "Adaptive ultra-short-term wind power prediction based on risk assessment," CSEE Journal of Power and Energy Systems, vol. 2, no. 3, pp. 59-64, 2016.
[5] S. Kulkarni and H.-P. Huang, "Changes in surface wind speed over North America from CMIP5 model projections and implications for wind energy," Advances in Meteorology, vol. 2014, Article ID 292768, 10 pages, 2014.

[6] K. Mohammadi, S. Shamshirband, D. Petković, and H. Khorasanizadeh, "Determining the most important variables for diffuse solar radiation prediction using adaptive neuro-fuzzy methodology; case study: city of Kerman, Iran," Renewable and Sustainable Energy Reviews, vol. 53, pp. 1570-1579, 2016.

[7] J. W. Taylor, P. E. McSharry, and R. Buizza, "Wind power density forecasting using ensemble predictions and time series models," IEEE Transactions on Energy Conversion, vol. 24, no. 3, pp. 775$782,2009$.

[8] C. Wan, Z. Xu, P. Pinson, Z. Y. Dong, and K. P. Wong, "Optimal prediction intervals of wind power generation," IEEE Transactions on Power Systems, vol. 29, no. 3, pp. 1166-1174, 2014.

[9] E.-M. Giannakopoulou and R. Nhili, "WRF model methodology for offshore wind energy applications," Advances in Meteorology, vol. 2014, Article ID 319819, 14 pages, 2014.

[10] B. Doucoure, K. Agbossou, and A. Cardenas, “Time series prediction using artificial wavelet neural network and multiresolution analysis: application to wind speed data," Renewable Energy, vol. 92, pp. 202-211, 2016.

[11] J. Yan, K. Li, E. Bai, Z. Yang, and A. Foley, "Time series wind power forecasting based on variant Gaussian Process and TLBO," Neurocomputing, vol. 189, pp. 135-144, 2016.

[12] J. Yan, Y. Liu, S. Han, Y. Wang, and S. Feng, "Reviews on uncertainty analysis of wind power forecasting," Renewable and Sustainable Energy Reviews, vol. 52, pp. 1322-1330, 2015.

[13] F. Cassola and M. Burlando, "Wind speed and wind energy forecast through Kalman filtering of numerical weather prediction model output," Applied Energy, vol. 99, pp. 154-166, 2012.

[14] H. Wei, H. Shao, and X. Deng, "Using a model structure selection technique to forecast short-term wind speed for a wind power plant in North China," Journal of Energy Engineering, vol. 142, no. 1, Article ID 04015005, 2016.

[15] D. Kukolj and E. Levi, "Identification of complex systems based on neural and Takagi-Sugeno fuzzy model," IEEE Transactions on Systems, Man, and Cybernetics, Part B: Cybernetics, vol. 34, no. 1, pp. 272-282, 2004.

[16] Y. Noorollahi, M. A. Jokar, and A. Kalhor, "Using artificial neural networks for temporal and spatial wind speed forecasting in Iran," Energy Conversion and Management, vol. 115, pp. 17-25, 2016.

[17] R. Ata, "Artificial neural networks applications in wind energy systems: a review," Renewable and Sustainable Energy Reviews, vol. 49, pp. 534-562, 2015.

[18] M. Saberivahidaval and S. Hajjam, "Comparison between performances of different neural networks for wind speed forecasting in Payam airport, Iran," Environmental Progress and Sustainable Energy, vol. 34, no. 4, pp. 1191-1196, 2015.

[19] H. Liu, H.-Q. Tian, Y.-F. Li, and L. Zhang, "Comparison of four Adaboost algorithm based artificial neural networks in wind speed predictions," Energy Conversion and Management, vol. 92, pp. 67-81, 2015.

[20] F. Cui, X. Deng, and H. Shao, "Short-term wind speed forecasting using the wavelet decomposition and AdaBoost technique in wind farm of East China," IET Generation, Transmission \& Distribution, vol. 10, no. 11, pp. 2585-2592, 2016.

[21] M. A. Mohandes, T. O. Halawani, S. Rehman, and A. A. Hussain, "Support vector machines for wind speed prediction," Renewable Energy, vol. 29, no. 6, pp. 939-947, 2004. 
[22] Q. Wu and C. Peng, "Wind power generation forecasting using least squares support vector machine combined with ensemble empirical mode decomposition, principal component analysis and a bat algorithm," Energies, vol. 9, no. 4, p. 261, 2016.

[23] J. Wang and J. Hu, "A robust combination approach for shortterm wind speed forecasting and analysis-combination of the ARIMA (Autoregressive Integrated Moving Average), ELM (Extreme Learning Machine), SVM (Support Vector Machine) and LSSVM (Least Square SVM) forecasts using a GPR (Gaussian Process Regression) model," Energy, vol. 93, pp. 41-56, 2015.

[24] X. Yuan, C. Chen, Y. Yuan, Y. Huang, and Q. Tan, "Short-term wind power prediction based on LSSVM-GSA model," Energy Conversion and Management, vol. 101, pp. 393-401, 2015.

[25] L. Yang, M. He, J. Zhang, and V. Vittal, "Support-vectormachine-enhanced markov model for short-term wind power forecast," IEEE Transactions on Sustainable Energy, vol. 6, no. 3, pp. 791-799, 2015.

[26] Y. Zhang, K. Liu, L. Qin, and X. An, "Deterministic and probabilistic interval prediction for short-term wind power generation based on variational mode decomposition and machine learning methods," Energy Conversion and Management, vol. 112, pp. 208-219, 2016.

[27] A. Kavousi-Fard, A. Khosravi, and S. Nahavandi, "A new fuzzybased combined prediction interval for wind power forecasting," IEEE Transactions on Power Systems, vol. 31, no. 1, pp. 1826, 2016.

[28] Z. Zhang, Y. Song, F. Liu, and J. Liu, "Daily average wind power interval forecasts based on an optimal adaptive-network-based fuzzy inference system and singular spectrum analysis," Sustainability, vol. 8, no. 2, p. 125, 2016.

[29] E. Álvarez-Miranda, C. Campos-Valdés, and C. Rahmann, "Two-stage robust UC including a novel scenario-based uncertainty model for wind power applications," Energy Conversion and Management, vol. 101, pp. 94-105, 2015.

[30] R. Juban, H. Ohlsson, M. Maasoumy, L. Poirier, and J. Z. Kolter, "A multiple quantile regression approach to the wind, solar, and price tracks of GEFCom2014," International Journal of Forecasting, vol. 32, no. 3, pp. 1094-1102, 2016.

[31] J. B. Bremnes, "Probabilistic wind power forecasts using local quantile regression," Wind Energy, vol. 7, no. 1, pp. 47-54, 2004.

[32] H. A. Nielsen, H. Madsen, and T. S. Nielsen, "Using quanti le regression to extend an existing wind power forecasting system With probabilistic forecasts," Wind Energy, vol. 9, no. 1-2, pp. 95-108, 2006.

[33] C. Guan, P. B. Luh, L. D. Michel, and Z. Chi, "Hybrid Kalman filters for very short-term load forecasting and prediction interval estimation," IEEE Transactions on Power Systems, vol. 28, no. 4, pp. 3806-3817, 2013.

[34] C. Guan, P. B. Luh, L. D. Michel, M. A. Coolbeth, and P. B. Friedland, "Hybrid Kalman algorithms for very short-term load forecasting and confidence interval estimation," in Proceedings of the IEEE Power and Energy Society General Meeting (PES '10), Minneapolis, Minn, USA, July 2010.

[35] A. Khosravi, S. Nahavandi, and D. Creighton, "Prediction intervals for short-term wind farm power generation forecasts," IEEE Transactions on Sustainable Energy, vol. 4, no. 3, pp. 602610, 2013.

[36] W. Charytoniuk and J. Niebrzydowski, "Confidence interval construction for load forecast," Electric Power Systems Research, vol. 48, no. 2, pp. 97-103, 1998.
[37] P. Pinson, "Wind energy: forecasting challenges for its operational management," Statistical Science, vol. 28, no. 4, pp. 564585, 2013.

[38] P. Pinson, C. Chevallier, and G. N. Kariniotakis, "Trading wind generation from short-term probabilistic forecasts of wind power," IEEE Transactions on Power Systems, vol. 22, no. 3, pp. 1148-1156, 2007.

[39] S.-W. Fei and Y. He, "Wind speed prediction using the hybrid model of wavelet decomposition and artificial bee colony algorithm-based relevance vector machine," International Journal of Electrical Power and Energy Systems, vol. 73, pp. 625-631, 2015.

[40] J. Wang, W. Zhang, Y. Li, J. Wang, and Z. Dang, "Forecasting wind speed using empirical mode decomposition and Elman neural network," Applied Soft Computing Journal, vol. 23, pp. 452-459, 2014.

[41] Y.-Y. Hong, T.-H. Yu, and C.-Y. Liu, "Hour-ahead wind speed and power forecasting using empirical mode decomposition," Energies, vol. 6, no. 12, pp. 6137-6152, 2013.

[42] Z. Wu and N. E. Huang, "Ensemble empirical mode decomposition: a noise-assisted data analysis method," Advances in Adaptive Data Analysis, vol. 1, no. 1, pp. 1-41, 2009.

[43] S. Wang, N. Zhang, L. Wu, and Y. Wang, "Wind speed forecasting based on the hybrid ensemble empirical mode decomposition and GA-BP neural network method," Renewable Energy, vol. 94, pp. 629-636, 2016.

[44] P. Nakaram and T. Leauhatong, "A new content-based medical image retrieval system based on wavelet transform and multidimensional wald-wolfowitz runs test," in Proceedings of the 5th Biomedical Engineering International Conference (BMEiCON '12), IEEE, Ubon Ratchathani, Thailand, December 2012.

[45] Y. Bao, H. Wang, and B. Wang, "Short-term wind power prediction using differential EMD and relevance vector machine," Neural Computing and Applications, vol. 25, no. 2, pp. 283-289, 2014.

[46] G. Sun, Y. Chen, Z. Wei, X. Li, and K. W. Cheung, "Day-ahead wind speed forecasting using relevance vector machine," Journal of Applied Mathematics, vol. 2014, Article ID 437592, 6 pages, 2014.

[47] H. Zang, Q. Xu, and H. Bian, "Generation of typical solar radiation data for different climates of China," Energy, vol. 38, no. 1, pp. 236-248, 2012.

[48] H. Bulut and O. Büyükalaca, "Simple model for the generation of daily global solar-radiation data in Turkey," Applied Energy, vol. 84, no. 5, pp. 477-491, 2007.

[49] H. Zang, Q. Xu, P. Du, and K. Ichiyanagi, "A modified method to generate typical meteorological years from the long-term weather database," International Journal of Photoenergy, vol. 2012, Article ID 538279, 9 pages, 2012.

[50] C. Wan, Z. Xu, and P. Pinson, "Direct interval forecasting of wind power," IEEE Transactions on Power Systems, vol. 28, no. 4, pp. 4877-4878, 2013. 

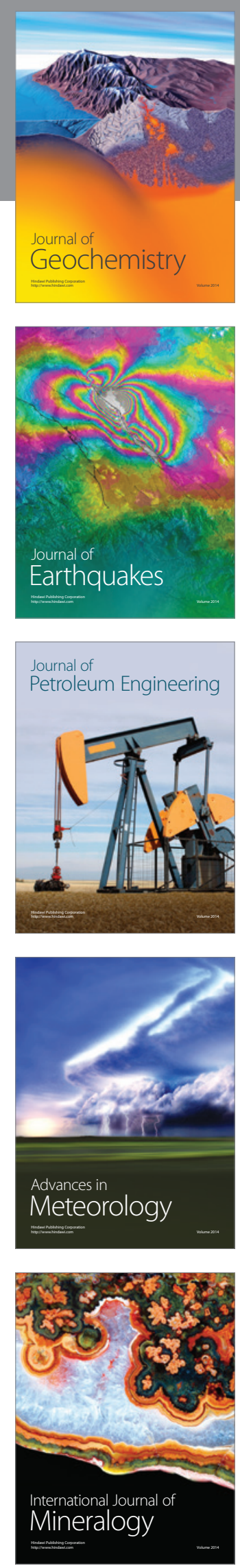
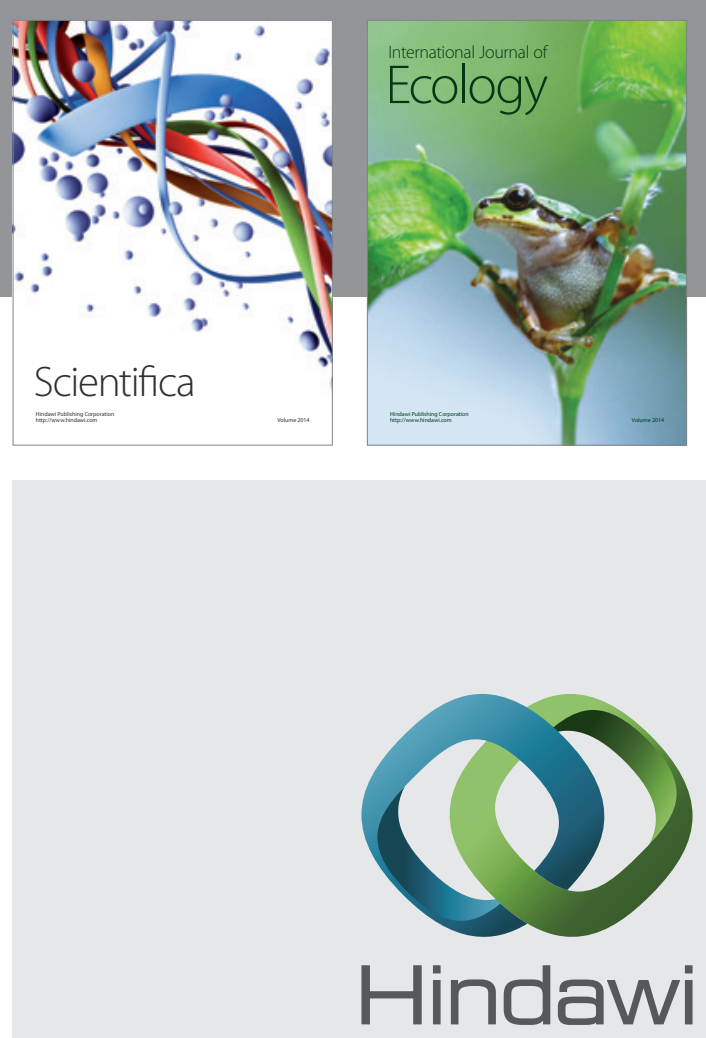

Submit your manuscripts at

http://www.hindawi.com
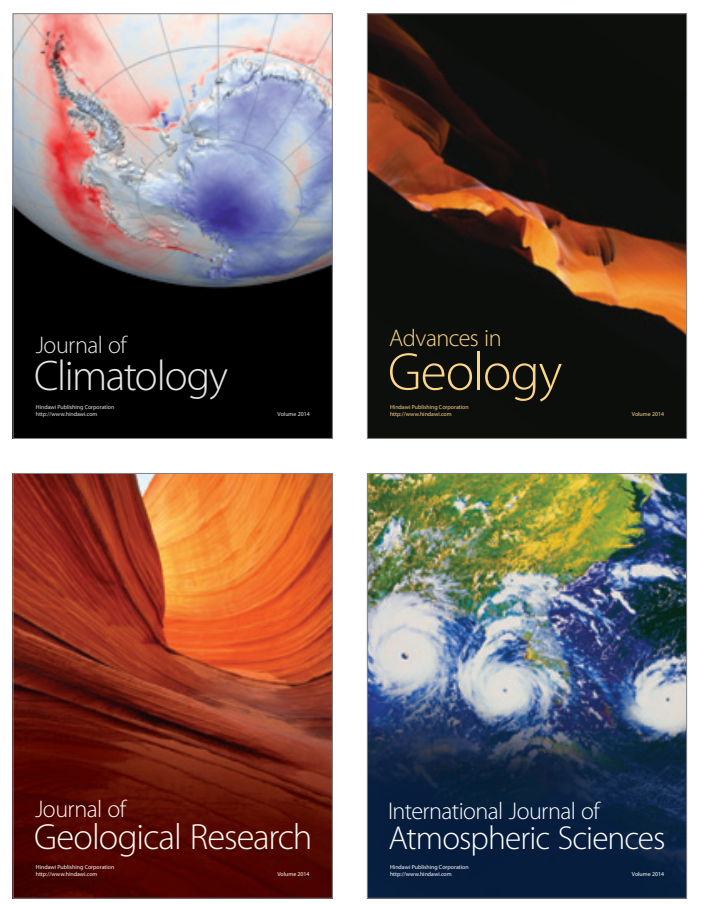

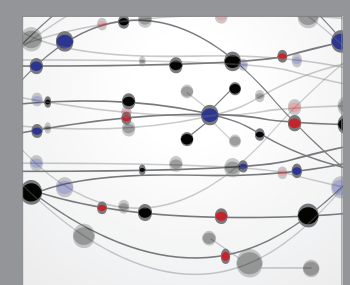

The Scientific

\section{World Journal}
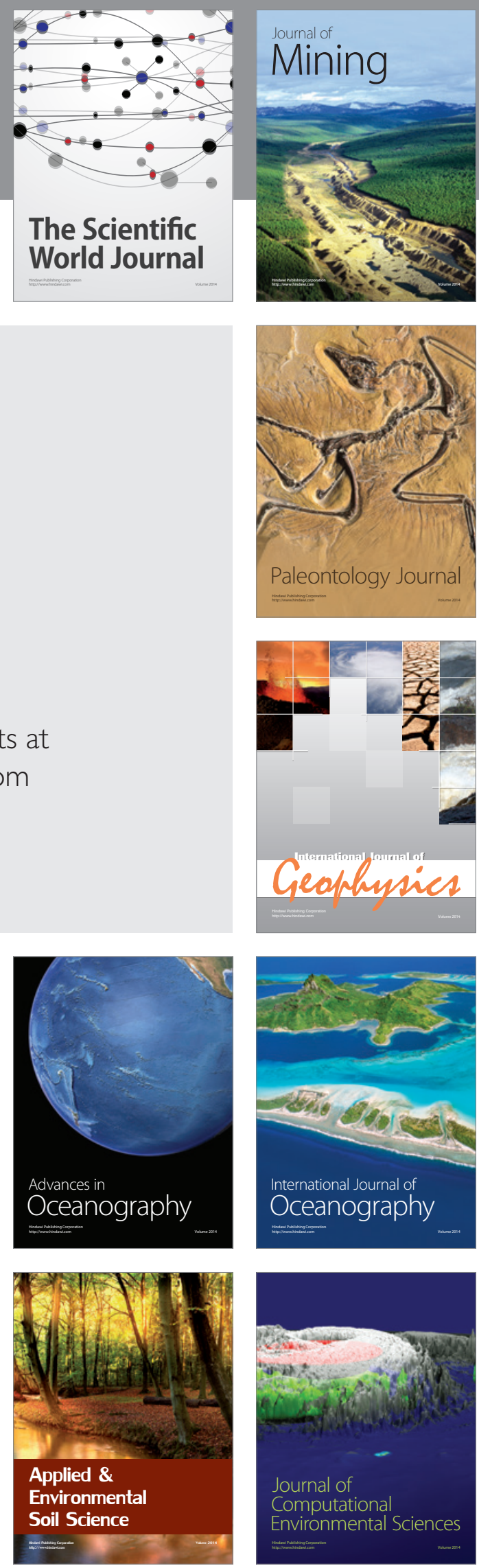